\title{
Direct Healthy Life Expectancy Estimates from Life Tables with a Sullivan Extension: The case of Brazil 2003
}

\author{
Yiannis Dimotikalis ${ }^{1}$ and Christos H Skiadas ${ }^{2}$ \\ ${ }^{1}$ Hellenic Mediterranean University, Department of Management Science and Technology, \\ Agios Nikolaos, Crete Greece (E-mail: jdim@ @mu.gr) \\ ${ }^{2}$ ManLab, Technical University of Crete, Chania, Crete, Greece \\ (E-mail: skiadas@cmsim.net)
}

\begin{abstract}
The Healthy Life Expectancy (HLE) in Brazil 2003 was estimated by Romero et al (2005) by using the Sullivan method and data from the World Health Survey carried out in Brazil in 2003. Here we use a Direct method to estimate the Healthy Life Years Lost (HLYL) and then the HLE. This is done after the analytic derivation of a more general model of survival-mortality and the estimation of a parameter $b_{x}$ related to the HLYL is followed by the formulation of a computer program providing results similar to those of the World Health Organization for the Healthy Life Expectancy (HALE) and the corresponding HLYL estimates. This program is an extension of classical life table including more columns to estimate the cumulative mortality, the average mortality, the person life years lost, and finally the HLYL parameter $b_{x}$. Even more, a further extension of the Excel program based on the Sullivan method provides estimates of the Healthy Life Expectancy at every year of the lifespan.
\end{abstract}

Key words: Healthy life expectancy, healthy life years lost, Sullivan.

\section{The HLYL Estimation Method}

The "Direct" Healthy Life Years Lost (HLYL) methodology Skiadas and Skiadas (2020 a,b,c) was based on averaging the Health State of a Population by taking into account the deaths/population from the mortality $m_{x}$ and the alive part of the population. A geometric approach of the methodology is presented in the following graph of mortality spaces where both mortality and survival are presented as corresponding areas. 


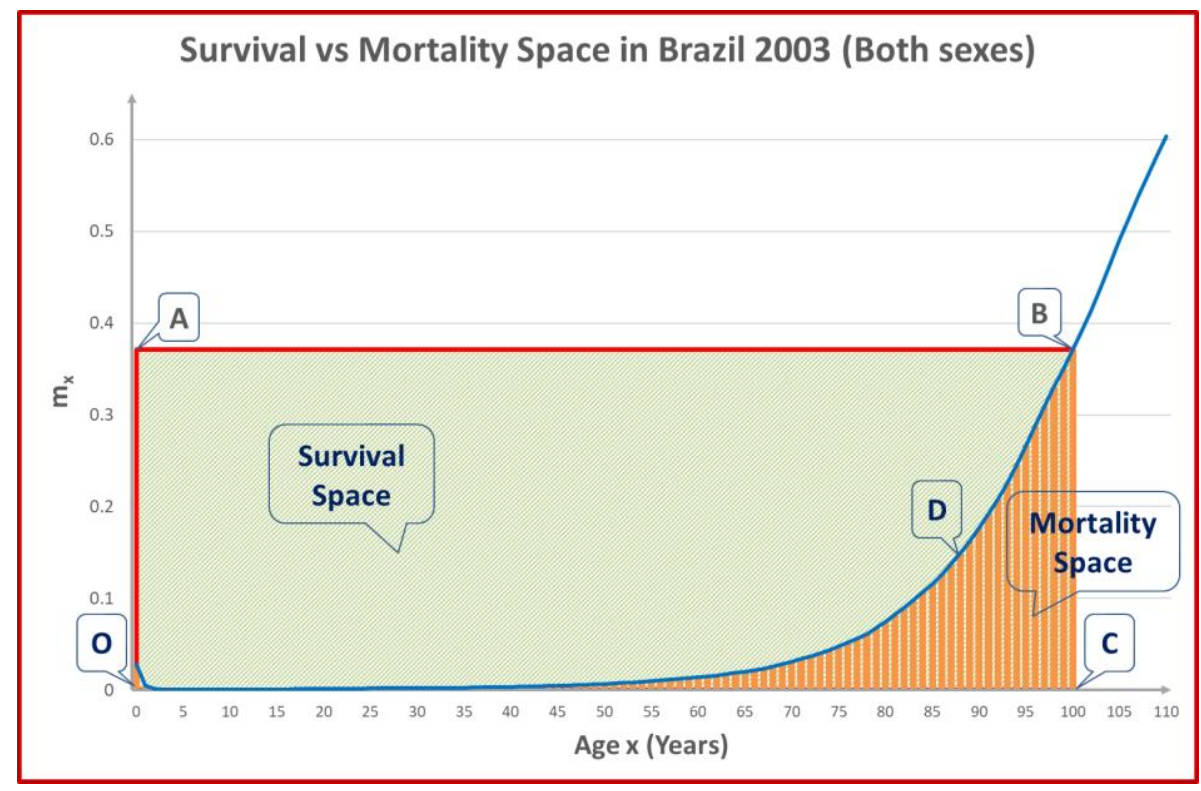

Fig. 1. Survival vs Mortality space graph

We use the Life Tables provided from the Human Mortality Database or any other related data base. Mortality is expressed by $m_{x}$ in these tables. In the above graph data from 2003 for the total population in Brazil was used. $m_{x}$ is shown as the blue exponential curve. The main forms of Life Tables start with $m_{x}$ and then the survival forms of the population are estimated. This methodology leads to the calculation of a probability measure termed as life expectancy at age $x$ or life expectancy at birth when considering the total life time. There are several differences between the graph with the survival space above and the survival curves methodology. First of all, the vertical axis in the Survival-Mortality Space (SMS) diagram is the probability $m_{x}$. Instead in the survival diagram the vertical axis represent population (usually it starts from 100.000 in most life tables and gradually slow down until the end). By the SMS diagram we have probability spaces for both survival and mortality. For the age $x$, the total space is (ABCOA) in the SMS diagrams, that is (OC).(BC) $=x m_{x}$. The mortality space is the sum $\mathrm{S}\left(m_{x}\right)$ while the survival space is $\left(x m_{x}-\mathrm{S}\left(m_{x}\right)\right)$. Accordingly, the important measure of the Health State is simply the fraction $(\mathrm{ABDOA}) /(\mathrm{BCODB})$. It is simpler to use the fraction $(\mathrm{ABCOA}) /(\mathrm{BCODB})=x m_{x} / \mathrm{S}\left(m_{x}\right)$ that can be estimated from $m x$ for every age $x$ of the population.

In modeling the healthy life years lost to disability some important issues should be realized. Mortality expressed by $m_{x}$ is important for modeling disability but more important is the cumulative mortality $S\left(m_{x}\right)$ which, as an additive process, is more convenient for the estimation of the healthy life years lived with disability and the deterioration process causing deaths. The estimates for this type of mortality are included in the term $b_{x} \mathrm{~S}\left(m_{x}\right)$.

The approach in previous publications (Skiadas and Skiadas (2018 a,b,c, 2020 a,b,c)) was to set a time-varying fraction $b_{x}$ for Health/Mortality of the form:

$$
b_{x}=\frac{x m_{x}}{\int_{0}^{x} m_{s} d s}
$$


This formula is immediately provided from figure 1 by considering the fraction:

$$
b_{x}=\frac{\text { Total Space }}{\text { Mortality Space }}=\frac{O A B C O}{O D B C O}=\frac{x m_{x}}{\int_{0}^{x} m_{s} d s}
$$

The main hypothesis is that the population involved in the deterioration process is a fraction of the total population at age $x$ determined by the level of mortality $m_{x}$ at age $x$. Accordingly, the mortality process will have two alternatives expressed by the simple equation:

$$
x m_{x}=b_{x} \int_{0}^{x} m_{s} d s \approx b_{x} \sum_{0}^{x} m_{x}
$$

Clearly when collecting data by asking people to respond to disability related questions, the answers is expected to be influenced from the previous experience from 0 to $x$ years of age including dead and alive. People comment on their disability in connection to their knowledge from the environment around. In a way, they respond after summarizing, averaging and assessing their knowledge in a way similar to the above methodology. Accordingly, it is not surprising to have similar results for Healthy Life Expectancy and Healthy Life Years Lost from both methods as the "Direct" and Health Adjusted Life Expectancy (HALE) applied by the World Health Organization (WHO). In the latter morbidity is adjusted by a specific parameter for every appropriate cause thus making the final estimate quite complicated. However, several decades of collecting data and applying data sets make HALE a classical tool for estimating the Health State of a Population. However, the HALE methodology turns only few decade years back as far as health data are already collected. For more unexplored health state periods or territories the Direct methodology is appropriate as far as Life Table Data exist.

\section{Program for the Estimates}

We have developed an Excel program for the Direct Estimates of $b_{x}$ which is provided free of charge. One version can be downloaded from the Demographics 2019 Workshop website at www.asmda.es . The program uses full life tables to provide the Healthy Life Year Lost estimator $b_{x}$ from the general equation form (1):

$$
b_{x}=\frac{x m_{x}}{\int_{0}^{x} m_{s} d s}
$$

The Cumulative Mortality Mx is given by

$$
M_{x}=\int_{0}^{x} m_{s} d s \approx \sum_{0}^{x}\left(\frac{d x}{l x}\right)
$$

Where $d x$ expresses the death population at age $x$ in the life tables and $l x$ is the remaining population at age $x$ in the same life tables. Note that the starting population at age $x=0$ is set at 100000 .

The average mortality $M x / x$ is estimated by

$$
\bar{M}_{x}=\frac{M_{x}}{x} \approx \frac{\sum_{0}^{x}\left(\frac{d x}{l x}\right)}{x}
$$


Then the Person Life Years Lost (PLYL) are provided by

$$
P L Y L=\frac{d x}{\bar{M}_{x}}=\frac{x d x}{M_{x}}
$$

The final estimate for $b_{x}$ is given by

$$
b_{x}=\frac{x m_{x}}{\int_{0}^{x} m_{s} d s} \approx \frac{P L Y L}{l x}=\frac{x d x}{l_{x} M_{x}}=\frac{x d x}{l_{x} \sum_{0}^{x}\left(\frac{d x}{l x}\right)}
$$

The methodology is presented in figure 2 that follows. The full life table from the HMD is followed by 4 more columns for the estimation of $b_{x}$. In the first, the cumulative mortality is estimated from $\mathrm{M}=\sum_{0}^{x} m_{x}$. The average mortality $(M / x)=\sum_{0}^{x} m_{x} / x$ is provided in the next column whereas the Person Life Years Lost (PLYL) $=\mathrm{x} d_{x} /\left(\sum_{0}^{x} m_{x}\right)$ are calculated in the next column. $d_{x}$ is provided by the column indicated by $d x$ in the life table. For this very important information, an interesting graph is provided. The graph follows a growth process until a high level and a decline in the remaining lifespan period. It the next column the Healthy Life Years Lost estimator $b_{x}$ is provided by dividing the PLYL by the $l x$ from the life table. The results are presented in an illustrative graph with the growing trend for $b_{x}$ to reach a maximum and a decline at higher ages. This high level can be also estimated by fitting the Weibull model (Weibull 1951, Skiadas 2020 a,b,c).

Another option added in this Excel is the estimates of the World Health Organization from 20002016 for Life Expectancy at birth and at 60 years of age for all the member countries whereas information for the Healthy Life Expectancy (HALE) at birth and at 60 years of age are provided for the years 2000, 2005, 2010, 2015 and 2016. We have added a small Table to present comparatively the WHO estimates with our estimates for the direct method. What is only needed after that is to copy and paste the life table and to select the name of the country in $11^{\text {th }}$ column and the gender (male, female or both sexes) in cell below in the Excel sheet. To avoid mistakes we have used the list of the WHO countries with their official names.

\section{A further HLE estimate based on the Sullivan method}

The classical Sullivan method is a standard tool to estimate the healthy life expectancy (see Sullivan, 1971 and Jagger, Van Oyen and Robine 1999). The simplicity of this method and the possibility to use it as a continuation or extension of the life table gave us the opportunity to add a Sullivan extension to the above-extended life table. By this extension, we have estimated the Healthy Life Years Lost (HLYL) for all the life span along with the Healthy Life Expectancy (HLE). The main part of the estimate is based on the proportion lived with disability. This is generated from the $b_{x}$ indicator from the previous columns multiplied with a discount Health Parameter. This is estimated directly from the program for our "direct" estimates and for WHO as well. For Eurostat estimates it is necessary to add the Healthy Life Expectancy at Birth in the appropriate box at the top of Figure 3. Another opportunity is by selecting the "Equal" option which automatically provides an estimate higher than HALE and closer to Eurostat. Every one of these options should be selected manually in the "Select" and "Parameter" places. The "Other" selection is set by the program user if needed. After selecting the appropriate Health Parameter, the next estimates follow automatically the Sullivan method, first for estimating the person-years 
lived without disability, then the total years lived without disability and finally the Disability Free Life Expectancy. The column of Health Life Years Lost follows.

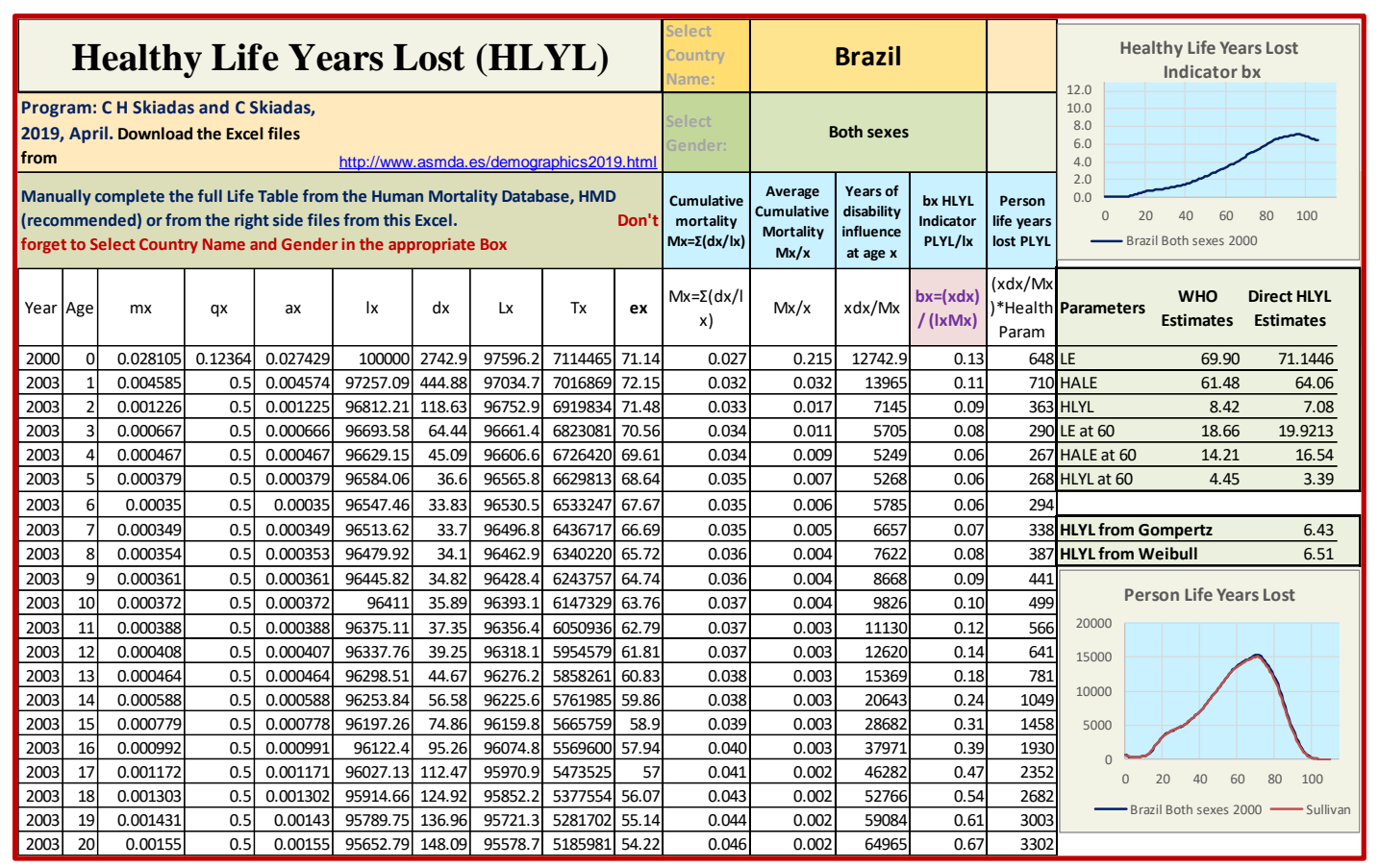

Fig. 2. The extended Life Table for the HLYL estimates. Download full program from http://www.smtda.net/demographics2020.html

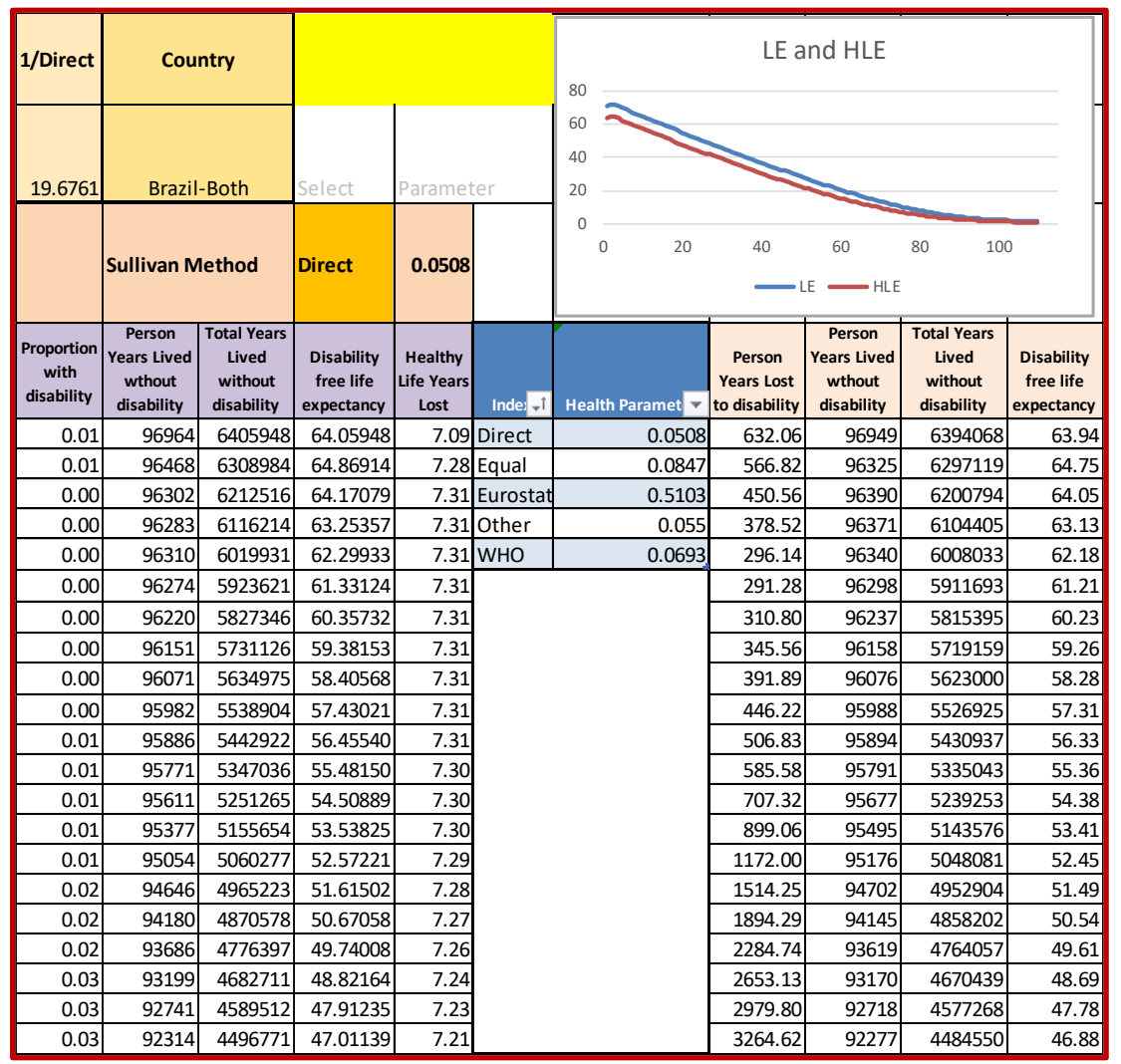

Fig. 3. The extended Life Table for the HLYL estimates with the Sullivan method 
An alternative method based on the Sullivan system is presented in the columns on the right-hand side of Figure 3. The estimates are based on the Person Years Lost to Disability and the direct estimates of the person-years lived without disability, the total years lived without disability and the Disability Free Life Expectancy with similar results with the previous approach. The estimated Healthy Life Years from two methods are presented in the appropriate graph of Figure 4a,b. The Health Parameters are estimated as follows:

Direct Parameter $=$ sumproduct $($ Lx.bx $) /($ Tx-(LE-HLYL).lx $)$

WHO Parameter $=$ sumproduct $($ Lx.bx)/(Tx-(HALE).lx $)$

Eurostat Parameter=sumproduct(Lx.bx)/(Tx-HLE.lx)

The inverse of the Equal Parameter corresponds to the Healthy Life Years Lost, that is:

HLYL=1/Equal

For the application for Brazil in 2003 the related estimates are included in the next Table:

\begin{tabular}{|c|c|c|c|c|c|c|c|c|c|}
\hline \multicolumn{7}{|c|}{ TABLE I: LE, HLE and HLYL for males, females and total in Brazil in 2003 } \\
\hline Method & $\begin{array}{c}\text { LE- } \\
\text { males }\end{array}$ & $\begin{array}{c}\text { HLE- } \\
\text { males }\end{array}$ & $\begin{array}{c}\text { HLYL- } \\
\text { males }\end{array}$ & $\begin{array}{c}\text { LE- } \\
\text { females }\end{array}$ & $\begin{array}{c}\text { HLE- } \\
\text { females }\end{array}$ & $\begin{array}{c}\text { HLYL- } \\
\text { females }\end{array}$ & $\begin{array}{c}\text { LE- } \\
\text { total }\end{array}$ & $\begin{array}{c}\text { HLE- } \\
\text { total }\end{array}$ & $\begin{array}{c}\text { HLYL- } \\
\text { total }\end{array}$ \\
\hline Direct & 67.3 & 60.9 & 6.5 & 75.0 & 67.3 & 7.7 & 71.2 & 64.1 & 7.1 \\
\hline $\begin{array}{c}\text { SKI-6 } \\
\text { Severe \& } \\
\text { Moderate }\end{array}$ & 67.3 & 52.7 & 14.6 & 75.0 & 58.6 & 16.4 & 71.2 & 55.8 & 15.3 \\
\hline $\begin{array}{c}\text { SKI-6 } \\
\text { Moderate }\end{array}$ & 67.3 & 61.4 & 5.9 & 75.0 & 66.7 & 8.3 & 71.2 & 64.4 & 6.7 \\
\hline $\begin{array}{c}\text { SKI-6 } \\
\text { Severe }\end{array}$ & 67.3 & 58.5 & 8.8 & 75.0 & 66.9 & 8.1 & 71.2 & 62.5 & 8.5 \\
\hline
\end{tabular}

Clearly the estimates for the HLE and the HLYL for males and females are similar for the Direct and the SKI-6 method. The latter is based on the model proposed by J. Janssen and Ch. Skiadas in 1995. As it is also found from the estimate based on the SKI-6 program (see Figure 6) presented in previous publications, both estimates (Direct and SKI-6) are close to Severe disability cases. To this end, the Direct and SKI-6 estimates refer to an important part of the life span with the life years lost to disability governing the development of everyday life.

Our Direct method, in addition to estimating the Healthy Life Expectancy, has the advantage of providing the Healthy Life Years Lost at every year of age via the $b_{x}$ parameter as is illustrated in Figure 4. Males and females show similar behavior for the age period 0-50 years of age with the exception of the years from 17-30 where an excess of life years lost to disability appear with the form of a higher $b_{x}$ for males than for females. After 50 years of age, the $b_{x}$ for females becomes higher with a maximum level at 95 years of age $(b=7.74)$ with a decline for the higher age years. For males, the maximum is at 95 years $(b=6.49)$ with a decline at higher ages. 

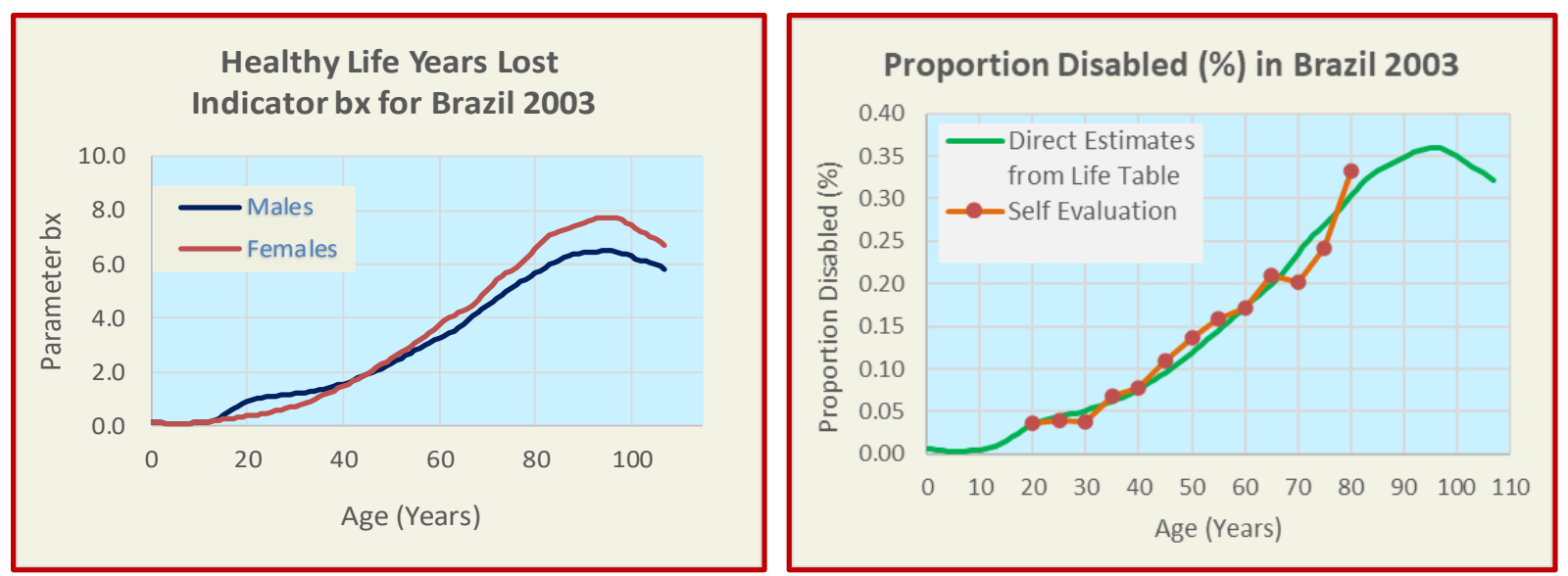

Fig. 4a,b. Healthy Life Years Lost for males and females (a) and total \% Disabled (b) in Brazil in 2003

Romero et al (2005) explored the Healthy Life Expectancy in Brazil in 2003 by applying the Sullivan method. The data collected by the 2003 survey. Figure $4 \mathrm{~b}$ illustrates the proportion of disabled persons estimated by Romero et al from the survey disability data (red curve) while the green curve represents our estimate with the "Direct" method from the complete 2003 Brazilian life table. Both methods provide similar results though the direct method explore the disability in more details.

The Person Life Years Lost for males and females in Brazil in 2003 as constructed from Figure 3 data in the Excel program are illustrated in Figure 5a. The highest level for males is at 68 years of age while an excess of mortality is at 17-30 years. Females present a maximum mortality level at 72 years of age. Both males and females follow a declining process after the maximum level. The results for the total population with both Direct and Self-evaluation methods appear in Figure 5b. The self-evaluation estimates come from Romero et al paper (Table 2) after appropriate calculations.
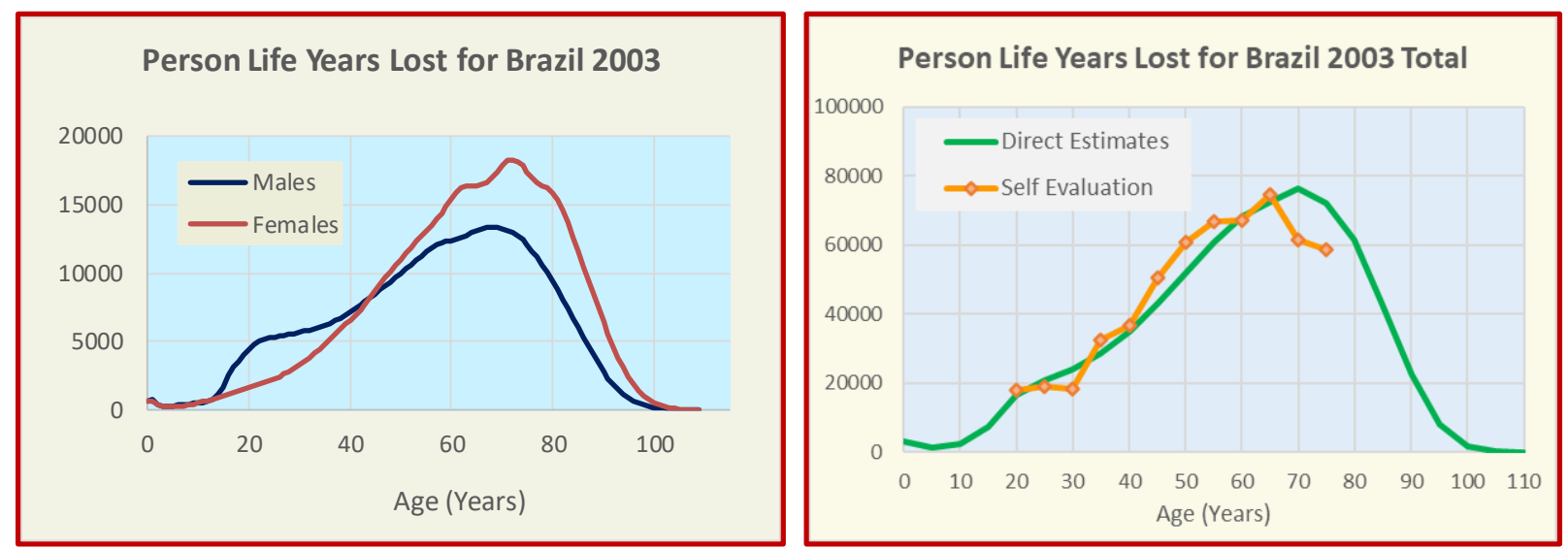

Fig. 5a,b. Person Life Years Lost for males and females (a) and total population (b) in Brazil in 2003. 


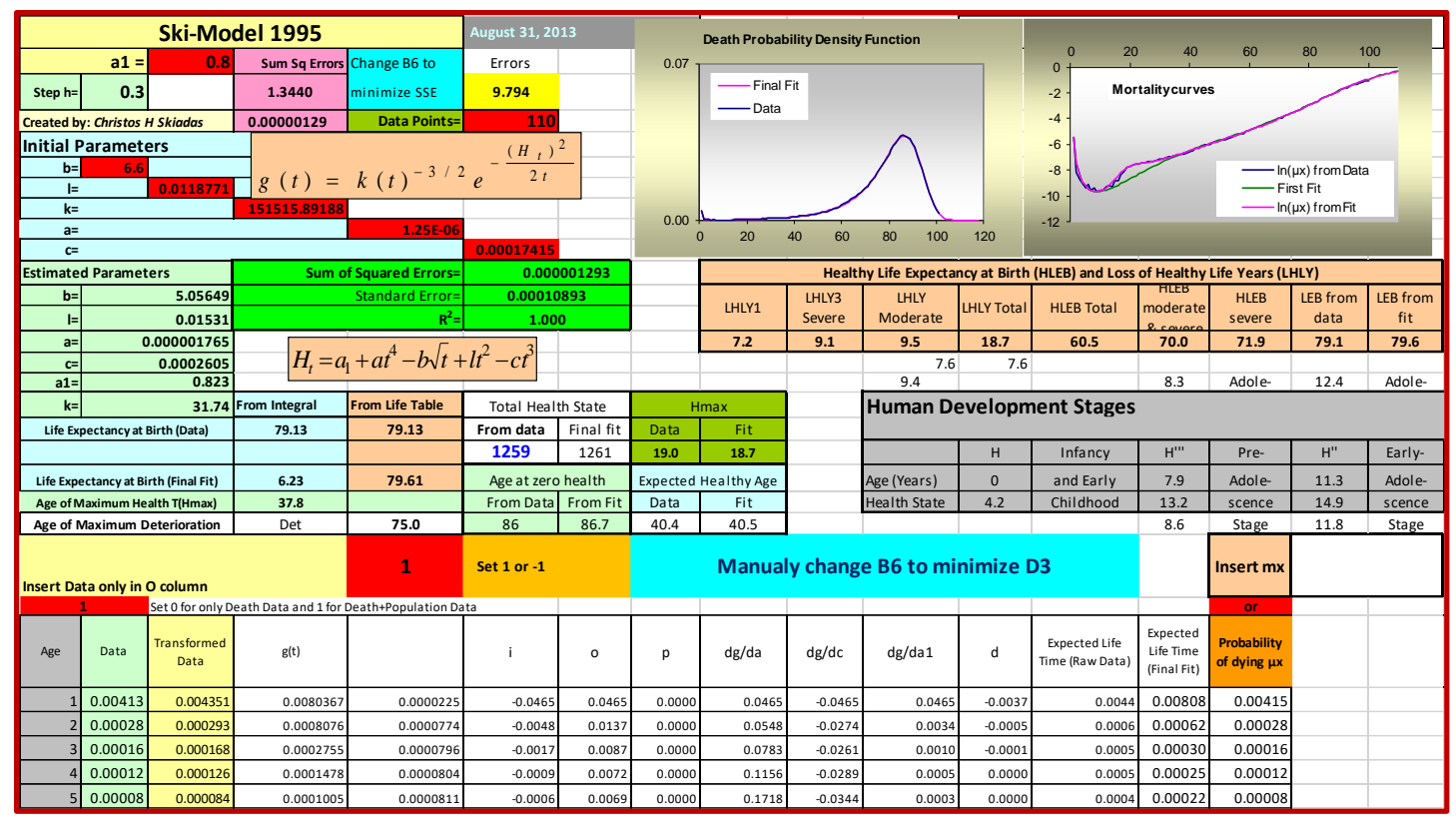

Fig. 6. The main view of the SKI-6 Excel Program and the Healthy Life Years Lost estimates. Download from: http://www.smtda.net/demographics2020.html

\section{Conclusions and Further Study}

We have provided an analytic explanation of the behavior of a parameter $b_{x}$ related to the healthy life years lost. We have also presented an analytic formulation for the observations made along the development of appropriate extensions of the classical life tables in order to give a valuable tool for estimating the Healthy Life Years Lost. We have also demonstrated that the results found for the general HLYL parameter that we proposed provide results similar to those provided by the Healthy Adjusted Life Expectancy and the corresponding HLYL estimates. An analytic derivation of the mathematical formulas is presented along with an easy to apply Excel program. A further extension of the Excel program based on the Sullivan method provides estimates of the Healthy Life Expectancy at every year of the lifespan by a Direct method. Estimates for Brazil in 2003 are presented. The latest versions of this program appear in the Demographics2020 Workshop website at http://www.smtda.net/demographics2020.html .

\section{References}

Jagger, C., Van Oyen, H. and Robine, J. M. (1999). Health Expectancy Calculation by the Sullivan Method: A Practical Guide.

Janssen, J., \& Skiadas, C. H. (1995). Dynamic modelling of life-table data. Applied Stochastic Models and Data Analysis, 11(1), 35-49.

Romero, D.E., Leite, I.C., Szwarcwald, C. L. (2005). Healthy life expectancy in Brazil: applying the Sullivan method. SciELO - Scientific Electronic Library Online. Cad. Saúde Pública 21 (suppl 1). https://doi.org/10.1590/S0102-311X2005000700002 
Skiadas, C.H. and Skiadas, C. (2018a). Exploring the Health State of a Population by Dynamic Modeling Methods. The Springer Series on Demographic Methods and Population Analysis 45, Springer, Chum, Switzerland. https://doi.org/10.1007/978-3-319-65142-2

Skiadas, C.H. and Skiadas, C. (2018b). The Health-Mortality Approach in Estimating the Healthy Life Years Lost Compared to the Global Burden of Disease Studies and Applications in World, USA and Japan. In Exploring the Health State of a Population by Dynamic Modeling Methods. The Springer Series on Demographic Methods and Population Analysis 45, Springer, Chum, Switzerland, pp 67-124. https://doi.org/10.1007/978-3-319-65142-2_4

Skiadas, C.H. and Skiadas, C. (2018c). Demography and Health Issues: Population Aging, Mortality and Data Analysis. The Springer Series on Demographic Methods and Population Analysis 46. Springer, Chum, Switzerland. https://doi.org/10.1007/978-3-319-76002-5

Skiadas C.H., Skiadas C. (2020a). Relation of the Weibull Shape Parameter with the Healthy Life Years Lost Estimates: Analytical Derivation and Estimation from an Extended Life Table. In: Skiadas C.H., Skiadas C. (eds) Demography of Population Health, Aging and Health Expenditures. The Springer Series on Demographic Methods and Population Analysis, vol 50. Springer, Cham. https://doi.org/10.1007/978-3-030-44695-6_2

Skiadas C.H., Skiadas C. (2020b). Direct Healthy Life Expectancy Estimates from Life Tables with a Sullivan Extension. Bridging the Gap Between HALE and Eurostat Estimates. In: Skiadas C.H., Skiadas C. (eds) Demography of Population Health, Aging and Health Expenditures. The Springer Series on Demographic Methods and Population Analysis, vol 50. Springer, Cham. https://doi.org/10.1007/978-3-030-44695-6_3

Skiadas C. H., Skiadas C. (2020c). How the unsolved problem of finding the Healthy Life Expectancy (HLE) in the far past was resolved: The case of Sweden (1751-2016) with forecasts to 2060 and comparisons with HALE. https://doi.org/10.31235/osf.io/akf8v

Sullivan, D. F. (1971). "A single index of mortality and morbidity." Health Services Mental Health Administration Health Reports 86: 347-354.

Weibull, W. (1951). A statistical distribution function of wide applicability. Journal of Applied Mechanics 18, 3, 293-297.

WHO Life Expectancy and Healthy Life Expectancy Data provided in Excel. http://apps.who.int/gho/athena/data/GHO/WHOSIS_000001,WHOSIS_000015,WHOSIS_00000 2,WHOSIS_000007?filter=COUNTRY:*\&format=xml\&profile=excel 